Carine Tarazi, MA, is an Assistant Editor for Pediatric Research in Boston, Massachusetts, USA. Margie Skeer, ScD, MPH, MSW, served as a Guest Editor for this special issue. Dr Skeer is Assistant Professor of Public Health and Community Medicine at Tufts University. Her research focuses on adolescent substance misuse and sexual risk prevention, both from epidemiologic and intervention-development perspectives. Kevin Fiscella, MD, MPH, served as a Guest Editor for this special issue. Dr Fiscella is Tenured Professor of Family Medicine, Public Health Sciences and Community Health at the University of Rochester Medical Center. Dr Fiscella's research focuses on health and health care disparities, particularly practical strategies to improve health equity. Stephanie Dean, MBA, is Managing Editor of Pediatric Research and is based out of editorial office in The Woodlands, Texas. OlafDammann, $M D$, served as a Guest Editor for this special issue. Dr Dammann is a Professor of Public Health and Community Medicine, Pediatrics, and Ophthalmology at Tufts University School of Medicine in Boston, Massachusetts, USA, as well as Professor of Perinatal Neuroepidemiology at Hannover Medical School, Hannover, Germany. His research interests include the elucidation of risk factors for brain damage and retinopathy in preterm newborns, the theory of risk and causation in biomedical and public health research, and the development of computational chronic disease models.

\title{
Everything is connected: social determinants of pediatric health and disease
}

\author{
Carine Tarazi ${ }^{1}$, Margie Skeer ${ }^{2}$, Kevin Fiscella ${ }^{3,4,5}$, Stephanie Dean ${ }^{1}$ and Olaf Dammann ${ }^{2,6}$
}

W hile basic science is the primary focus of Pediatric Research, the balance of articles has varied from issue to issue in recent years. In 2015, the Journal published papers about cognitive impairment, emotional and behavioral problems, cardiovascular risk factors, lead exposure, prenatal opioid exposure, and obesity, among other areas of focus that have distinctly social aspects. Rarely, however, are these social aspects mentioned: biological science mostly deals with influences within an organism, separate from the workings of the society it lives in. For the most part, considerations such as socioeconomic status, race, and education are considered outof-bounds. There is a word limit, after all.

The 2016 Review issue of Pediatric Research acknowledges the elephant in the room. In short, context matters. Just as "the neck bone's connected to the back bone," the health and safety of a child are connected to the physical and psychosocial nurturance of his or her environment. From the moment of conception, outside forces impact a child's life. This recognition is of paramount importance to physicians and researchers alike.

Malnutrition is due to lack of vitamins and minerals, but is that deficiency a result of lack of resources, lack of care, lack of information, or a combination of these problems? Similarly, chronic stress from a myriad of contextual factors can have a lasting impact on child development. The biology may be the same in every case, but the root causes often differ widely.

We are pleased to be presenting articles that incorporate many disciplines. Our authors come not only from the medical field but also the specialties of public health, social work, psychiatry, psychology, bioethics, and epidemiology. The reviews in this issue discuss the roles played by socioeconomic disparities, caregivers, and early life experiences in pediatrics. These topics intersect in most of the papers, but the reviews are categorized here by their most noteworthy feature.

Socioeconomics and race play perhaps the largest role in the type of care one receives. With this in mind, the first two reviews concern epigenetics: Rubin explores how environmental stresses can lead to biological change in both mothers and children; and Burris et al. examine racial disparities in preterm

\footnotetext{
IInternational Pediatric Research Foundation, The Woodlands, Texas; ${ }^{2}$ Department of Public Health and Community Medicine, Tufts University School of Medicine Boston, Massachusetts; ${ }^{3}$ Rochester Center to Improve Communication in Health, Rochester, New York; ${ }^{4}$ Greater Rochester Practice Research Network, Rochester, New York; ${ }^{5}$ Westside Community Health Center, Rochester, New York; ${ }^{6}$ Perinatal Neuroepidemiology Unit, Hannover Medical School, Hannover, Germany. Correspondence: Carine Tarazi (carine.tarazi@pedres.org)
} 


\section{Introduction | Taraziet al.}

birth that could potentially be attributed to social and environmental exposures. With the goal of targeted intervention, Lorch and Enlow highlight disparities in perinatal outcomes depending on race and ethnicity. Hurt and Betancourt review how early socioeconomic status is seen as having an effect on brain structure and language outcomes. With an emphasis on pediatric kidney diseases, Sereni et al. evaluate the need for illness management in low-to-middle income countries. Marcin et al. review the use of telemedicine in rural communities.

The critical role parents or primary caregivers play in child health is reflected in the second set of articles. The first, by Renk et al., reviews the efficacy of parenting interventions for substance-involved mothers and fathers. The next, by Beal et al., focuses on children in nonparental care, their well-being, and the adversities they are likely to face. Children in medical foster care is the subject of a paper by Seltzer et al. that discusses the risks confronted by children of parents who cannot afford to pay for appropriate care. Weiss-Laxer et al. write about models for treating and preventing maternal depression.
The topic of early life experiences is inextricably linked to childcare but is more concerned with development. A review by Campbell summarizes the determinants of child obesity and another by Mitchell et al. concerns early-life effects on epigenetic patterns. A review by Guinosso et al. looks at child cognitive development in adverse circumstances and evaluates intervention strategies. Oral et al. review trauma-informed care for children. There is also a Commentary by Krugman asking why the topic of child abuse is not more widely discussed within the pediatric research community, and a response by Sege regarding the urgent need for more efforts to prevent child abuse and neglect.

Pediatric care exceeds the realm of biological science, as evidenced by the longstanding conversation around the impact of nature vs. nurture on development. Although basic science research values quantifiable data, social determinants also play a critical role in children's security and well-being. We hope this collection encourages further research on questions crucial to child health and social equity. 